\title{
LANGUAGE DEVELOPMENT IN TYPICALLY DEVELOPING CHILDREN AND CHILDREN WITH DEVELOPMENTAL DEFICITS: A CONCISE QUALITATIVE REVIEW
}

\author{
Mila Vulchanova, Valentin Vulchanov \\ Language Acquisition and Language Processing Lab \\ Norwegian University of Science \& Technology - Norway
}

\begin{abstract}
:
The current review provides a concise qualitative overview of how language develops in typically developing children in comparison to developmental deficits which adversely affect language, with a focus on language impairment (Developmental Language Disorder) and autism spectrum disorder (ASD). The review traces the main stages in language acquisition and observed trajectories in typical children and children with developmental deficits. The main factors which impact on language development are highlighted and seen in the context of language outcomes. The review covers research across languages and cultures, and across populations of speakers.
\end{abstract}

Keywords: language development, typically developing children, developmental deficits, language competence, developmental trajectories, factors in language development

\section{Typical language development}

\subsection{Typical language development and developmental deficits}

Language development is a long process characterized by dynamic changes in children's communicative competences and skills at different levels of language structure. Typical language development progresses gradually from speech perception to learning to associate objects and situations in the world with their linguistic labels, and further on to understanding and producing sentences of increasing complexity, and, ultimately, longer pieces of discourse and interaction mediated by language (Karmiloff, Karmiloff-Smith 2001).

Despite the observed variation in individual rates of development (Bretherton et al. 1983; Rescorla 1984; Huttenlocher et al. 1991), most children achieve the same 
milestones (e.g., first words, first two-word combinations) roughly around the same age. In some cases, however, developmental disorders disrupt the process of typical development by selectively affecting both the process of language learning and its outcomes. Among developmental deficits which affect language, the most prevalent one is Language Impairment (also labelled Developmental Language Disorder). Its prevalence is estimated at 6-7\% (Boyle et al. 1996; Tomblin et al. 1997), but prevalence may vary depending on cut-off thresholds and instruments/criteria used in the screening or diagnosis. Language Impairment (LI) adversely affects the child's ability to acquire language leading to poor outcomes in receptive and/or expressive language skills. Affected children are likely to have language problems enduring into middle childhood and beyond, with a significant impact on everyday social interactions or educational progress (Bishop et al. 2017). While early delay in language may be transient, around half of the children with language delay may experience persistent problems with language later on (Bishop, Edmundson 1987; Rescorla, Schwartz 1990; Rescorla et al. 1993; Thal, Bates 1988). Autism spectrum disorder (ASD) is a pervasive neuro-developmental condition characterized by a constellation of earlyappearing social communication deficits and repetitive sensory-motor behaviours. Like LI, the prevalence of autism may vary depending on the availability of screening tools, referral practice and cut-off points. Current prevalence estimates for the USA are 1 in every 54 children (Maenner et al. 2016), while for the UK the prevalence estimate is $1 \%$. (Baron-Cohen et al. 2009). ASD is associated with a strong genetic component as well as other causes (Lord et al. 2018). The range of structural language skills on the autism spectrum is highly varied, whereby some affected children hardly produce any speech (minimally verbal), while others may have adequate structural language competence (highly-verbal) (Tager-Flusberg 2006). For this reason, according to the prevalent current view reflected in DSM-5 (American Psychiatric Association 2013), the presence of language impairment is seen as a co-morbid condition, rather than the primary impairment in autism. Still, even highly verbal individuals with autism have problems with discourse comprehension and figurative language processing, often described as impairment in pragmatic language (Tager-Flusberg 2006; Vulchanova et al. 2015; Chahboun et al. 2016; Vulchanova et al. 2019). Both LI and ASD impact adversely on oral language outcomes in affected children. To the extent that oral language is the primary mode of language acquisition and use, these two populations will be of main interest for the current review.

In the first stages of language acquisition, very young infants are busy processing the sounds of language. Research provides evidence that this process starts already in the pre-natal stage while the foetus is in the womb, and already in the last trimester of pregnancy, the hearing system is sufficiently well developed to appreciate a variety of sounds, including language. More importantly, already at this stage, the foetus can discriminate between different types of sounds, and can isolate language as a special type, where sequences of sounds potentially have a special value (Mehler et al. 1988). Needless to say, during the pre-natal stage, the quality of sound is very different from sound that travels through air: the yet unborn baby is surrounded by liquid (i.e. the amniotic fluid) and sound is quite muffled. Research, however, documents that newly 
born infants are sensitive to voice quality and can distinguish between the mother's voice and other female voices. They also show a specific preference for the mother's voice as heard in the womb over the mother's voice heard after birth, as well as a strong preference for the mother's voice over other female voices. Importantly, young infants prefer their own mother's voice provided the mother speaks with a normal intonation (Mehler et al. 1978). There is a natural explanation for this phenomenon, which is the result of rich exposure to language produced by the mother both prior to birth and after birth. As a matter of fact, language experience through exposure to language is by far the most important among environmental factors in language development (Gathercole, Hoff 2007). Also, the preference for the mother's voice is explained through its high bonding value in the first months and years after birth.

\subsection{Speech perception in infancy}

During early speech perception, while distinguishing between different languages and especially their intonational properties, infants gradually develop a preference for their native language, and by the time they are 9 months old, sensitivity to other languages and their sound systems declines dramatically. This very early sensitivity window to speech closes before the first year of life, and the loss of sensitivity to the sound systems of other languages provides some explanation to the anecdotally attested difficulty in acquiring the pronunciation of foreign languages later in life. Interestingly enough, infants raised bilingually seem to retain sensitivity to more than one language beyond this time window, as documented in experimental studies on Spanish/Catalan and English/French (Sebastian Galles et al. 2012; Weikum et al. 2007). This is one of many advantages that have been found for early bilinguals.

It has been shown that infant phonetic discrimination is initially language universal, however, a decline in this ability is observed between the $6^{\text {th }}$ and the $12^{\text {th }}$ month after birth. It has been argued that this decline in early sensitivity to universal phonological properties of language is associated, and correlates, with nativelanguage phonetic learning (Kuhl, Conboy, Padden et al., 2005). In a seminal study, Kuhl and colleagues (2005) followed longitudinally a cohort of infants from the time they were 7-month-old to 30 months of age. In that study native language phonetic discrimination was a reliable predictor of later language skills in participating infants. Thus, better native-language discrimination at 7 months predicted accelerated later language abilities, whereas better nonnative-language discrimination at 7 months predicted reduced later language abilities when tested at 30 months.

The study by Kuhl et al. (2005) highlights the role of early speech perception as a predictor of later acquired language competences and skills. Thus, even though during early speech perception, the infant is primarily occupied with processing the sounds of language, without necessarily processing for meaning, early speech perception plays an important role for the rest of language acquisition - it provides a stepping stone for identifying potential words in the speech stream, later to learn them as the labels of objects and situations the infants are surrounded by in their ambient environment. Being able to segment the speech stream into smaller units 
(e.g., possible words) is an important achievement which can be compared to learning a foreign language - originally it all merges into a never ending and unsegmentable blur of sounds to the inexperienced ear. Infants gradually learn to break this code and start identifying frequently and stably occurring sequences, which actually correspond to words in the native language (Jusczyk 1997). There are many cues that infants can rely on in this process. Across languages, stress is an important clue where a word ends and where a new one starts, thus helping in establishing word boundaries. Some languages rely on tones, which distinguish between words, where the same sequence of sounds produced with two different tones may mean two very different things. This distinction can be found even in less exotic languages, such as Norwegian (Nilsen 2000).

Other cues for word learning may lie in the so-called phonotactic rules of the language. For each language there are namely legal and illegal combinations of sounds. For instance, in Spanish the combination of ," " and „p“ is illegal at the beginning of words. Thus, you need to add „e" before them to make the word pronounceable in Spanish (Spain - Espagna). Phonotactics is encoded in the orthography of the language at hand and thus plays an important role in the acquisition of literacy. In recent research we document that bilingual exposure to orthotactically dissimilar languages may provide an advantage in the process of acquiring literacy skills (Borragan et al. 2020). We tested two groups of bilingual children, Spanish/Catalan (two languages which are orthotactically similar) and Spanish-Basque (orthotactically different), and compared the bilingual groups to monolingual children. The SpanishCatalan bilingual children performed similarly to the monolingual controls and were much better on recognition of words that followed the orthotactic rules of their native languages (i.e., legal words), while the Spanish-Basque bilingual children showed equal performance in learning illegal and legal patterns. These findings suggest that bilinguals with dissimilar languages are more flexible in also considering new and potentially possible orthotactic patterns. This pattern of performance in the Spanish-Basque bilinguals is reminiscent of the attested sensitivity to universal phonological properties in the first months after birth (Kuhl et al. 2005) and suggests that the window of learning phonotactic/orthotactic rules is still open in that group of bilingual children. Furthermore, we demonstrate that word learning is not affected by bilingualism as such, but rather depends on the bilinguals' specific linguistic experience at this age.

While speech perception helps in word learning, early speech production is quite different in nature. Here, the infant needs to train its vocal apparatus in order to master and perfect producing the specific sound sequences of their native language. The individual sounds of each language require a lot of effort to produce by featuring subtle variations in the quality of the sound and place of articulation. Thus ,p“ and „,“" are distinguished solely on the basis of „,voiced“ quality. Giving motor instructions to your lips and tongue is quite a feat, and infants start practicing already in the second half of their first year of life by engaging in the activity typically referred to as babbling. Here they produce combinations of vowels and consonants (baba, dada), 
and often more complex sequences often resembling existing words in the language. Parents would sometimes proudly take this as word production, however, it is the first steps at mastering speech production.

\subsection{Words, words, words}

Early word learning is a very intriguing process. It requires a lot of previously acquired skills, such as identifying the sound pattern that corresponds to a specific word, and as such, largely depends on early speech perception. A second important step is the ability to identify what aspect of the world this sequence of sounds possibly describes. Is it an object (a dog/cat/cup), a situation (running/eating), a property (nice/ red/stupid), or some relation between objects (the spoon is IN the cup)? The infinite possibilities in how words map onto objects and events out in the world has become known as the „Gavagai“" problem and can be traced back to a philosophical mental experiment first described by Quine (Quine 1960).

There are many cues that infants can follow in the process of word learning. One important cue is the ability to follow the caregiver's attention, a phenomenon known as joint attention. This ability may be impaired in children with autism, and is often assumed as one of the causes of the delay in their language development. The ability to form categories (e.g., perceiving similarity between objects of the same type) is another crucial competence. Furthermore, research suggests that often categorization and word learning go hand in hand and assist each other, and children have been shown to more readily form categories in the presence of language labels (e.g., in experiments with invented words (i.e. pseudo-words) vs. pro-forms, e.g. „Show me the blick“" vs. "Show me this one"). Thus, it is impossible to state whether categorization leads word learning or whether word knowledge is a necessary prerequisite for early categorization. Interesting evidence comes from work conducted by Linda Smith and her colleagues, where studies have shown the importance of object shape in the process of word learning (Yee et al. 2012). Interestingly, changes in the visual recognition of objects precede the observed shape bias in noun learning. From that work, it becomes clear that shape supersedes colour, texture and other object properties in the process of word learning. In addition, this work shows that the visual affordances of objects, and object shape, in particular, provide a cognitive backdrop for the acquisition of language labels.

Studies addressing early word learning document that elaborate processes are already in place. Infants and toddlers respond to sound similarity between words suggesting a growing vocabulary, and later on become sensitive also to semantic similarities between the meanings of words (Mani, Plunkett 2010). We have evidence that the infant „mental“ lexicon (i.e, all the words that the infant can recognize and produce) gradually grows in size and density, with an increasing number of new connections between newly learned words and already existing word knowledge (Mani et al. 2012). A recent theory suggests that children actually learn the words they want to learn (Mani, Ackerman 2018), and this might explain, in part, the documented differences in vocabulary knowledge between boys and girls. 


\subsection{Language structure}

While words are important in order for the child to be able to name objects, acquiring the hidden rules of grammar of the native language is what ultimately leads to understanding complex utterances, in both oral communication and when reading texts. Grammar competence lies at the heart of later academic skills, which require access to content in written form (e.g., in textbooks or on-line learning materials). As such, it is also a major predictor of how well children will understand texts at school, and will communicate their thoughts both orally and in a written form. Grammar competence in the native language has also been shown to determine comprehension in a second language, e.g. a language the child learns later at school. In a study of a large sample of 10-year old Norwegian children we documented reliable correlations between phonological memory and working memory and overall language skills in both Norwegian (first language) and English (an early second language) (Vulchanova et al. 2014). More importantly, English sentence comprehension in our participants was predicted directly by their first language (Norwegian) grammar/syntax competence as measured on a comprehensive language test battery. At the early stages of language acquisition, vocabulary and grammar appear to be inseparable, and early word knowledge has been found to both correlate with, and predict, later grammar skills around the second year of life (Bates, Goodman 1997). Later, after the first school years, word knowledge, grammar and discourse skills emerge as independent components of the language faculty, and can be identified as separate dimensions (LARC 2015). This simply means that they can be studied in the child independently, and are less interrelated in middle childhood and adolescence.

\subsection{Pragmatic language development}

Pragmatic language is a cover term applying to language competences and skills which operate at a level beyond syntax. These are typically related to discourse comprehension, the ability to understand language in context, but also the interpretation of non-literal figurative language, such as jokes, irony, indirect requests, idioms, metaphors. The reason why these skills emerge later is not only because they are scaffolded by earlier skills, such as adequate vocabulary knowledge and advanced syntax competence. In addition, their emergence is associated with the acquisition of foundational literacy and, through literacy, with richer exposure to written language, and, thus, exposure to more and more varied registers of the native language (Levorato et al. 2004; Cain et al. 2008; Vulchanova et al. 2015).

Figurative language displays exponential non-linear development between the pre-school years and middle childhood. Research in idiom comprehension and processing in children (Benneli et al. 2006, Levorato, Cacciari 2002), has further suggested that the ability to work with figurative language (including idioms) correlates with age and years of schooling and is linked to other linguistic abilities, such as meta-linguistic awareness, and the ability to infer from context (Bernicot et al. 2007). 
In a study designed to test for the first time idiomatic knowledge in younger children (6-7 years), and older children (9-10 years) with Bulgarian as a first language (L1), as compared to adults, we show that, while idiom comprehension is scarce among the younger group, children aged 9-10 display advanced linguistic skills and are capable of providing elaborate definitions and synonyms for the target items (Vulchanova et al. 2011). Our study also shows that children only resort to literal interpretations in the cases when they do not know the idiom, and that some of their „,incorrect" responses reflect an embodied perspective on language (Simmons et al. 2003). Our results indicate that different types of idiomatic expressions present different types of challenges for the learner and are acquired differently. For instance, idioms like „Да се измокря до кости“ (get soaking wet (lit. „get wet to the bones"), „Да си спася кожата“ (save one's skin) and „Хлопа му дъската“ (have a screw loose) were familiar also to the younger participants, and received close to ceiling performance in the older children. Such idioms are biologically motivated in that they build on analogies involving parts of the body, and, as such lexicalize and encode the basis of human experience. There is strong evidence that many of them are shared across cultures, and we find almost word-by-word equivalents across the Indo-European languages, but also Finnish (cf. Penttilä et al. 1998). Biologically motivated idioms belong to the category of early acquired non-literal expressions and their acquisition plateaus around 11-12 years. In contrast, culturally-based idioms „Гладна мечка хоро не играе“ (hungry bellies have no ears (lit. ,a hungry bear would not dance“); „Свалям картите на масата“ (lay/put your cards on the table) which reflect cultural and often idiosyncratic aspects of the language, are acquired gradually and along the life-span. One cultural idiom, „Работи, без да подвие крак“('work without rest' (,work without twisting/bending a leg") was particularly challenging for the child participants. We hypothesize that this is the case because the expression is based on an obsolete (idiosyncratic) lexicalization pattern. From the point of view of the modern language, the verb подвие ('twist/bend') is odd in the context of the direct object крак ('leg'). The expression, as a whole, refers to a sitting posture where your legs are placed under your body and are bent. However, it is archaic and not transparent to the modern speaker. Furthermore, it is highly likely that obsolete expressions of this type are less frequent in the input children are currently exposed to, thus reducing opportunities for becoming familiar with the expression.

\subsection{Gesture and language}

Gesture and speech form a unitary communication system whereby they are closely aligned both semantically and temporally (McNeill 1992; Cooperrider, Goldin-Meadow 2017). Gesture comprehension, and specifically, gesture production, are inherently linked to emerging communicative skills in the child, and are early precursors of later language skills (Butterworth 2003; Iverson, Goldin-Meadow 2005). The distinctive use of hand for communicative purposes signals infants' socio-cognitive development (Tomasello et al. 2007) and their readiness for spoken language acquisition (for a meta-analysis see Colonnesi et al. 2010). As a matter of 
fact, the cognitive underpinnings of pointing are at the very heart of communication development and make apparent the link between gestures, cognition and language (Kita 2003). Among early gestures, pointing (deictic) gestures have been shown to be closely linked to later language development. In contrast to other deictic gestures (i.e. showing and giving), pointing allows for reference-making without necessarily establishing contact with the referent. Pointing production thus implies the acquisition of the cognitive skills necessary to go beyond pure object manipulation and engage in symbolic reference from a distance. Pointing is also the only gesture type that has been shown to reliably predict language in both typically developing children (TD) and children with autism (Özçalışkan et al. 2016; Ramos-Cabo et al. 2019 for a review).

Research in developmental deficits documents that gesture is often impaired in children with autism and Developmental Language Disorder (DLD) (BaronCohen 1989; Goodhart, Baron-Cohen 1993; Camaioni et al. 2003). In a recent study we document that deictic gestures are not only quantitatively affected, but also qualitatively impaired in children with an autism diagnosis in comparison to typically developing children and children at high risk for autism (Ramos-Cabo et al. 2021). Thus, gesture production can be used as an early marker of atypical communicative function.

\section{Factors in language development}

\subsection{Biological and cognitive factors}

There are broadly two types of factors that impact on the process of acquiring the native language: biological and environmental. Among the biological factors, we can mention brain maturation, which determines brain plasticity. This is also known as the sensitive period for language acquisition, a favourable window in the life of the child, during which language can be acquired optimally. Even though it is uncertain whether this period has a clear terminus, i.e. a point beyond which language acquisition cannot be successful and cannot reach ultimate attainment of language competence, there is evidence that the ability to acquire language declines with age. A recent large-scale study of both native speakers and second language learners of English in the USA has established that indeed the language learning ability declines in late adolescence (Hartshorne et al. 2018). This decline can be partly explained in terms of the gradual loss of brain plasticity, meaning that, as adults, we are less open to learning new skills. Other accounts would rather focus on the adult cognitive status, which differs from that of children, and the fact that children have fewer, and possibly, less sophisticated, cognitive resources available to them. As a result, they can learn language better than adults, an account come to be known as Elissa Newport's less-is-more hypothesis (https://www.psychologicalscience.org/ publications/observer/25at25/elissa-l-newport.html). 
Other biological factors that might play a role in language development are gender differences (also observed in brain maturation).

Among cognitive factors, phonological memory and working memory determine how well the child can store and manipulate sounds and sound segments, a core mechanism which underlies language learning. Often, this ability can be impaired in developmental deficits (e.g., developmental language deficit/language impairment, reading impairment), which, in turn, affects the course and trajectory of learning.

\subsection{Environmental factors}

By far the most important environmental factor in language development is the language ecology of the child, the language it is exposed to. This is language the child hears from caregivers (parents, grand-parents), teachers in pre-school, and peers (children of the same age as the child). This exposure makes it possible for the child to benefit from the variation of the language used, whether in terms of dialects, idiolects or generational differences in language use. It is exactly rich variation in the language input that ultimately leads to successful generalization across contexts and acquisition of the main aspects of language: from sound, to word to grammar, and the rules that underlie their use. In addition, social factors, such as early child-adult interaction, parental education and involvement with the child have been shown to play an important role.

Bilingualism provides important evidence of the role of exposure to language input. In an increasingly global world children are raised in bilingual, and often, multilingual environments. Children who are exposed to two or more languages from early on (around birth and during the first years of life) are thus likely to acquire both languages without effort and develop balanced competences in both languages (Werker, Byers-Heinlein 2008; Klein et al. 2014). However, the extent to which bilingual individuals attain competence in the languages they are exposed to depends on the complex interplay of multiple factors, including age of exposure to the language in question (the biological constraints on language acquisition), quantity and quality of input (language environment), and language activity and use (e.g., how often and for what purposes the language is used) (Genesee, Nicoladis 2007; Grosjean 2001).

Importantly, the biological and environmental factors in language development work in synergy, and successful language acquisition is brought about through the dynamic interaction between nature and nurture. This means that genes do not operate in a deterministic way, and that learning itself impacts on brain development in the course of this interaction.

At present, a new important factor is already affecting human culture, behaviour and cognition, and is changing our children's lives and childhood dramatically. New technologies create environments that could alter how we process information, the degree to which we take risks, how we socialize and empathize with others and even, how we view our own identity. Parents experience that toddlers can handle digital tools with a level of sophistication that they can only envy. We are immersed in a 'digital ecology' increasingly populated by devices that are both tools at our disposal and 
interactive agents with a degree of autonomy. While software development in both the entertainment and learning domain is advancing at a fast and unprecedented rate, and schools are spontaneously adopting digital solutions for the acquisition of academic skills, such as literacy, at present, there is no adequate research base documenting the effects of digital tools on language or other learning outcomes in young children. Neither are current software solutions based on developmental, educational or psycholinguistic principles. Thus, we are facing the consequences of what educational and cognition researchers have called ,a vast unplanned experiment“ (Hirsh-Pasek et al. 2015). An open question is to what extent the increasingly digital environments will equip young children with an adequate type of input concerning language acquisition, and whether this exposure will have positive or negative consequences (e.g., in comparison to interaction with human caregivers) (Vulchanova et al. 2017).

It has been maintained that the nature of the language to be acquired does not play a decisive role in the process of language acquisition and its successful outcome. Thus, whether the child is exposed to a richly inflected language (such as e.g., Finnish) or a language with poor morphology (e.g., English), is generally not expected to play a role in success in acquisition and whether the child achieves ultimate attainment or not. Interestingly, however, research has documented that, despite being more challenging, richly inflected languages may trigger more efficient (and faster) acquisition of grammatical categories. A study comparing 1001 English-speaking children and 386 Italian-speaking children between age 1;6 and 2;6 demonstrates large cross-linguistic differences in the amount of morphology that has been acquired in children matched for vocabulary size, as well as small, but intriguing differences in the shape of the growth curve for grammatical function words (Caselli et al. 1999). These results indicate that children acquiring a morphologically complex language (e.g., Italian) possess more advanced grammar competence in comparison to children of the same age exposed to a morphologically impoverished language, like English. This appears to be a consequence of the richer cues that Italian provides for the learner and can be explained in terms of the Competition Model (Bates, McWhinney 1982), whereby cue availability and reliability play a role in determining the order of acquisition of grammatical structures. Such cues may, in turn, recruit and more strongly activate the use of Statistical Learning, a powerful experience-dependent mechanism heavily implicated in the process of language acquisition (Saffran et al. 1996; Saffran 2003). Interestingly, there is emerging evidence that some languages may prove more challenging for the language learner. A recent conceptual review suggests that, unlike closely related languages, such as e.g., Norwegian, Danish has an unusually reduced phonetic structure, which seemingly delays Danish-learning children in several aspects of their language acquisition (Trecca et al. (in press)a; Trecca et al. (in press)b).

\subsection{Other factors}

Parents often wonder whether instruction plays a role in native language development. Based on the evidence we have from research, and numerous observations, it can be safely stated that infants and young children do not respond 
to correction or instruction on part of the parents. Thus, oral language development depends on the subtle weighting of, and synergy between, the biological and environmental factors, but not on explicit instruction. Importantly, however, the acquisition of literacy in the native language requires a certain deal of instruction, especially in the first years of school. Instruction or modelling behavior can also be seen as beneficial in the provision of intervention for children with developmental deficits. Often during intervention, the speech and language therapist provides an explicit model, which the child can follow, e.g., asking specific types of questions or comparing words and their meaning or model sentences in order to practice different types of constructions (a practice often called reinforcement).

Another factor, which often comes to mind in the context of language development, is motivation. Given rich exposure to language in the environment and sufficient interaction with both parents and peers, it can be imagined that motivation has no role. Some researchers have emphasized the social and communicative value of language as encouraging a type of inherent motivation to learn language. Indeed, for the developing child, becoming aware of the power of language in both establishing and maintaining social bonds, is an important incentive to learn to use that code. In addition, new research suggests that there might be an inherent reward in learning language. A word learning study by Antoni Rodriguez-Fornells and his colleagues (Ripolles et al. 2014) provides evidence that successful word acquisition activates brain areas supporting reward mechanisms in the brain. This comes to suggest that there may be an even deeper inherent drive to acquire language, also seen from an evolutionary perspective.

\section{Language development in developmental deficits}

Children with developmental deficits often experience problems with language. In children with language impairment (also currently known as Developmental Language Disorder) biological factors conspire to compromise the very process of language development, ultimately leading to impaired outcomes. It has thus been suggested that inadequate language skills are the consequence of (impaired) language learning (Bishop 2013). Such children will experience problems in producing speech and word learning, but most commonly the problems are present in core aspects of grammar, such as word inflections and sentence structure. Whereas the most pronounced problems in children on the autism spectrum are in social communication and interactions, as well as the presence of repetitive behaviours and lack of flexibility, most children with an autism diagnosis will also experience problems with aspects of language. While for children on the low end of the spectrum, the language problems may be pervasive, for children on the high end (often called high-functioning or highly-verbal), structural aspects of language will be largely intact. However, even for those children, subtle problems remain, such as e.g, in the understanding of nonliteral language found in idioms, metaphors, jokes, irony and humour (Vulchanova et al., 2015; Chahboun et al., 2016; Vulchanova et al, 2019). Whether these problems 
are related to the core deficit or to language competence is yet to be established, and the source of these problems is still open to debate and future research. Children with a reading problem will primarily have problems with the sound structure of the native (or other languages) and its mapping to orthography (the written form of the language). Some research provides evidence that there may be additional factors, such as, morphological skills, which impact on (impaired) reading, and this might also depend on the word structure of the language in question and how it is encoded in its orthography.

Children with language-related disorders often present with word learning problems (see Vulchanova et al. 2020 for a review). Such problems may affect either the acquisition of phonological aspects of the word (word form) or aspects of word meaning, depending on the type of deficit. Thus, children with DLD struggle with problems related to word form/morphology (Nation 2014), whereas the acquisition of word semantics is more challenging for children with autism (Norbury et al. 2010). Such dissociations in word learning, which lead to poor outcomes in word knowledge, provide corroboratory evidence of theoretical and psycholinguistic models of word knowledge (Levelt 1989) and representation (e.g., Perfetti's Lexical Quality Hypothesis - Perfetti, Hart 2002; Perfetti 2007).

In most cases of language-related developmental deficits, affected children will display a marked delay in the development of specific aspects of language compared to similarly aged children. Thus, an important task for parents is to closely observe how the child is acquiring the native language and seek advice or screening for language, if there is concern that the child is not developing on track. Also, sudden changes in the language trajectory (e.g., a „mute/silent" period or regression in previously attained language skills) should be cause for concern.

\section{Concluding remarks and aspects worthy of notice}

An important feature of typical language development is the well attested big individual variation in language skills in the course of development. This variation is the product of the biological and environmental forces that impact on language development and is quite natural. In a similar way, the child's receptive skills vastly outstrip her/his expressive skills, a phenomenon clearly observed in the early years. Thus, it takes almost a year before the infant starts producing language (mainly words), even though they are perfectly capable of understanding simple messages and instructions long before that. The asymmetry between comprehension and production is a well-established fact, and should not cause any concern, if observed. It deserves notice that in a global world, many children will be raised bilingually. Their language skills in both languages will largely depend on their language ecology (e.g., how often and for how long they are exposed to the languages they are acquiring; whether one of the languages is dominant or not; the family language practices). A crucial factor in bilingual language outcomes is the age of onset, meaning when the child starts learning the language. In the past there have been concerns among educators 
and parents that acquiring two languages simultaneously may cause a delay, even confusion in the child. However, research over the past 40 years provides ample evidence that bilingual children can succeed in acquiring both languages given optimal conditions: rich and varied exposure and early onset (Werker, Byers-Heinlein 2008). How well adults learn new languages will depend again on their learning environment, e.g., whether in a classroom or through immersion in the culture and the language. Yet, given that there is a sensitive period for language, we might expect that their language skills in that second language will not be optimal.

\section{References}

American Psychiatric Association 2013: Diagnostic and statistical manual of mental disorders (5th ed.). <https://doi.org/10.1176/appi.books.9780890425596>

Baron-Cohen 1989: Baron-Cohen, S. Perceptual role taking and protodeclarative pointing in autism. British Journal of Developmental Psychology, 7, 113-127.

Baron-Cohen et al. 2009: Baron-Cohen, S., Scott, F.J., Allison, C., Williams, J., Bolton, P., Matthews, F. E., Brayne C. Prevalence of autism-spectrum conditions: UK school-based population study. British Journal of Psychiatry, 194(6):500-9.

Simmons et al. 2003: Simmons, W. K., Barbey, A. K., Wilson, C. D. Grounding conceptual knowledge in modality-specific systems. Trends in Cognitive Sciences, 7, 2, 84-91.

Bates, Goodman 1997: Bates, E., Goodman, J. C. On the inseparability of grammar and the lexicon: Evidence from acquisition, aphasia, and real-time processing. Language and Cognitive Processes, 12(5/6), 507-584.

Bates, McWhinney 1982: Bates, E., McWhinney, B. Functionalist approaches to grammar. In: E. Wanner and L. Gleitman (eds.), Language Acquisition: The State of the Art. New York: Cambridge University Press.

Benneli et al. 2006: Benneli, B., Belacchi, C., Gini, G., \& Lugangeli, D. To define means to say what you know about things': the development of definitional skills as metalinguistic acquisition. Journal of Child Language, 33, 71-97.

Bernicot et al. 2007: Bernicot, J., Laval, V., Chaminaud, S. Nonliteral language forms in children: In what order are they acquired in pragmatics and metapragmatics? Journal of Pragmatics, 39, 2115-2132.

Bishop 2013: Bishop, D. Cerebral Asymmetry and Language Development: Cause, Correlate, or Consequence? Science, Vol. 340, Issue 6138, 1230531.

Bishop, Edmundson 1987: Bishop, D. V. M., Edmundson, A. Language-impaired 4-yearolds: distinguishing transient from persistent impairment. Journal of Speech and Hearing Disorders 51, 98-110.

Bishop et al. 2017: Bishop, D. V. M., Snowling, M. J., Thompson, P. A., Greenhalgh, T., the CATALISE-2 consortium. Phase 2 of CATALISE: a multinational and multidisciplinary Delphi consensus study of problems with language development: Terminology. Journal of Child Psychology and Psychiatry, 58(10), 1068-1080.

Borragan et al. 2020: Borragan, M., De Bruin, A., Havas, V., De Diego-Balaguer, R., Vulchanova, M., Vulchanov, V., Duñabeitia, J. Differences in word learning in children: Bilingualism or linguistic experience? Applied Psycholinguistics, 1-22.

Boyle et al. 1996: Boyle, J., Gillham, B., Smith N. Screening for early language delay in the 18-36 month age-range: the predictive validity of tests of production, and implications for practice. Child Language Teaching and Therapy, 12(2): 113-127. 
Bretherton et al. 1983: Bretherton, I., McNew, S., Snyder, L., Bates, E. Individual differences at 20 months: analytic and holistic strategies in language acquisition. Journal of Child Language 10, 293-320.

Butterworth 2003: Butterworth, G. \$Pointing is the royal road to language for babies“. In Pointing: Where language, culture, and cognition meet. ed. S. Kita (Mahwah, NJ: Lawrence Earlbaum Associates), 9-33.

Cain et al. 2008: Cain, K., Towse, A. S., Knight, R. S. The development of idiom comprehension: An investigation of semantic and contextual processing skills. Journal of Experimental Child Psychology, 102, 280-298.

Camaioni et al. 2003: Camaioni, L., Perucchini, P., Muratori, F., Parrini, B., Cesari, A. The communicative use of pointing in autism: developmental profile and factors related to change. European Psychiatry, 18, 6-12. <https://doi.org/10.1016/s0924-9338(02)00013-5>

Caselli et al. 1999: Caselli, C., Casadio, P. \& Bates, E. A comparison of the transition from first words to grammar in English and Italian. Journal of Child Language, 26 (1), 69 111. <https://doi.org/10.1017/S0305000998003687>

Chahboun et al. 2016: Chahboun, S., Vulchanov, V., Saldaña, D., Eshuis, H., \& Vulchanova, M. Can You Play with Fire and Not Hurt Yourself? A Comparative Study in Figurative Language Comprehension between Individuals with and without Autism Spectrum Disorder. PloS one, 11(12), e0168571. < https://doi.org/10.1371/journal.pone.0168571>

Colonnesi et al. 2010: Colonnesi, C., Stams, G. J. J. M., Koster, I., Noom, M. J. The relation between pointing and language development: a meta-analysis. Developmental Review 30, 352-366. < https://doi.org/10.1016/j.dr.2010.10.001>

Cooperrider, Goldin-Meadow 2017: Cooperrider, K. \& Goldin-Meadow, S. When Gesture Becomes Analogy. Topics in Cognitive Science, 9: 719-737. < https://doi.org/10.1111/ tops.12276>

Gathercole, Hoff 2007: Gathercole, V., \& Hoff, E. Input and the Acquisition of Language: Three Questions. In E. Hoff \& M. Shatz (Eds.), Blackwell handbooks of developmental psychology. Blackwell handbook of language development (pp. 107-127). Malden, Mass.: Blackwell. <https://doi.org/10.1002/9780470757833.ch6>

Genesee, Nicoladis 2007: Genesee, F. \& Nicoladis, E. Bilingual First Language Acquisition. In: Blackwell Handbook of Language Development (eds E. Hoff and M. Shatz). <https:// doi.org/10.1002/9780470757833.ch16>

Goodhart, Baron-Cohen 1993: Goodhart, F., Baron-Cohen, S. How many ways can the point be made? Evidence from children with and without autism. First Language, 13, 225-233.

Grosjean 2001: Grosjean, F. The bilingual's language modes. In Nicol, J. (Ed.). One Mind, Two Languages: Bilingual Language Processing (pp. 1-22). Oxford: Blackwell.

Hartshorne et al. 2018: Hartshorne, J., Tenenbaum, J., Pinker, S. A critical period for second language acquisition: Evidence from 2/3 million English speakers. Cognition, Volume $177,263-277$.

Hirsh-Pasek et al. 2015: Hirsh-Pasek, K., Zosh, J. M., Golinkoff, R. M., Gray, J. H., Robb, M. B., \& Kaufman, J. Putting Education in „Educational“ Apps: Lessons From the Science of Learning. Psychological Science in the Public Interest, 16(1), 3-34. <https:// doi.org/10.1177/1529100615569721>

Huttenlocher et al. 1991: Huttenlocher, J., Haight, W., Bryk, A., Seltzer, M., Lyons, T. Early vocabulary growth: relation to input and gender. Developmental Psychology 27, 236-248. 
Iverson, Goldin-Meadow 2005: Iverson, J. M., \& Goldin-Meadow, S. Gesture paves the way for language development. Psychological Science 16, 367-371. <https://doi. org/10.1111/j.0956-7976. 2005.01542.x>

Jusczyk 1997: Jusczyk, P. The Discovery of Spoken Language. MIT Press.

Karmiloff, Karmiloff-Smith 2001: Karmiloff, K. \& Karmiloff-Smith, A. Pathways to language: From fetus to adolescent. Cambridge, MA: Harvard University Press.

Kita 2003: Kita, S. Pointing. Where language, culture, and cognition meet. Mahwah, NJ: Lawrence Erlbaum.

Klein et al. 2014: Klein, D., Mok, K., Chen, J.K. \& Watkins, K. E. Age of language learning shapes brain structure: a cortical thickness study of bilingual and monolingual individuals. Brain and Language 131, 20-4. <https://doi.org/10.1016/j.bandl.2013.05.014.>

Kuhl et al. 2005: Kuhl, P.K., Conboy, B., Padden, D., Nelson, T. \& Pruitt, J. Early Speech Perception and Later Language Development: Implications for the „Critical Period“. Language Learning and Development, 1 (3 \& 4) (2005), 237-264.

Language and Reading Research Consortium 2015: The Dimensionality of Language Ability in Young Children. Child Development, 86, 1948-1965. <https://doi.org/10.1111/ cdev.12450>

Levelt 1989: Levelt, W.J.M. Speaking: From Intention to Articulation. MIT Press, Cambridge, MA.

Levorato, Cacciari 2002: Levorato, M., C., Cacciari, C.The creation of new figurative expressions: psycholinguistic evidence in Italian children, adolescents and adults. Journal of Child Language, 29, 127-150.

Levorato et al. 2004: Levorato, M. C., Nesi, B., Cacciari, C. Reading comprehension and understanding idiomatic expressions: A developmental study. Brain and Language, 91. 303-314.

Lord et al. 2018: Lord, C., Elsabbagh, M., Baird, G., Veenstra-Vanderweele, J. Autism spectrum disorder. Lancet (London, England), 392(10146), 508-520. <https://doi. org/10.1016/S0140-6736(18)31129-2>

Mani, Plunkett 2010: Mani, N., Plunkett, K. In the infant?s mind?s ear: Evidence for implicit naming in 18-month-olds. Psychological Science, 21, 908-13.

Mani et al. 2012: Mani, N., Durrant, S., Floccia, C. Activation of phonological and semantic codes in toddlers. Journal of Memory and Language 66, 612-22.

Mani, Ackermann 2018: Mani, N. \& Ackermann, L. Why Do Children Learn the Words They Do?. Child Dev Perspect, 12, 253-257. <https://doi.org/10.1111/cdep.12295>

Maenner et al. 2016: Maenner MJ, Shaw KA, Baio J. Prevalence of Autism Spectrum Disorder Among Children Aged 8 Years - Autism and Developmental Disabilities Monitoring Network, 11 Sites, United States. MMWR Surveillance Summary 2020;69(No. SS-4):112. < http://dx.doi.org/10.15585/mmwr.ss6904a1external icon>

McNeill 1992: McNeill, D. Hand and mind: What gestures reveal about thought. Chicago: University of Chicago Press.

Mehler et al. 1978: Mehler, J., Bertoncini, J., Barriere, M., \& Jassik-Gerschenfeld, D. Infant Recognition of Mother's Voice. Perception, 7(5), 491-497. <https://doi.org/10.1068/ p070491>

Mehler et al. 1988: Mehler, J., Jusczyk, P., Lambertz, G., Halsted, N., Bertoncini, J. \& AmielTison, C. A precursor of language acquisition in young infants. Cognition, 29, 143-178.

Nation 2014: Nation, K. Lexical learning and lexical processing in children with developmental language impairments. Philosophical Transactions of the Royal SocietyB 369: 20120387. 
Nilsen 2000: Nilsen, R. A. Tonale særtrekk i norske lavtonedialekter. Folkmålsstudier.

Norbury et al. 2010: Norbury, C.F., Griffiths, H., Nation, K. Sound before meaning: word learning in autistic disorders. Neuropsychologia 48, 4012-4019.

Quine 1960: Quine, W.V.O. Word and object. Cambridge, MA: MIT Press.

Penttilä et al. 1998: Penttilä, E., Nenonen, M., \& Niemi, J. Cultural and Biological Bases of Idioms: A Crosslinguistic Study. In J. Niemi et. all (Eds.), Language Contact, Variation, and Change. (Studies in Languages, 32, University of Joensuu), 234-245.

Perfetti 2007: Perfetti, Ch. Reading ability: Lexical quality to comprehension. Scientific Studies of Reading. 11 (4). 357-383.

Perfetti, Hart 2002: Perfetti, Ch., Hart, L. The lexical Quality Hypothesis. - In: Verhoeven, Elbro and Reitsma (eds.) Precursors of functional literacy 11, 67-86.

Ramos-Cabo et al. 2019: Ramos-Cabo, S., Vulchanov, V., Vulchanova, M. Gesture and Language Trajectories in Early Development: An Overview From the Autism Spectrum Disorder Perspective. Frontiers in Psychology, 10, 1211.

Ramos-Cabo et al. 2021: Ramos-Cabo, S., Vulchanov, V, Vulchanova, M. Different Ways of Making a Point: A Study of Gestural Communication in Typical and Atypical Early Development. Autism Research.

Rescorla 1984: Rescorla, L. Individual differences in early language development and their predictive significance. Acta Paedologica 1, 97-116.

Rescorla, Schwartz 1990: Rescorla, L., Schwartz, E. Outcome of toddlers with specific expressive language delay. Applied Psycholinguistics 11, 393-407.

Rescorla et al. 1993: Rescorla, L., Hadicke-Wiley, M., Escarce, E. Epidemiological investigation of expressive language delay at age two. First Language 13, 5-22.

Ripollés et al. 2014: Ripollés, P., Marco-Pallarés, J., Hielscher, U., Mestre-Missé, A., Tempelmann, C., Heinze, H. J., Rodríguez-Fornells, A. \& Noesselt, T. The Role of Reward in Word Learning and Its Implications for Language Acquisition. Current Biology, Volume 24, Issue 21, 2606-2611.

Saffran 2003: Saffran, J. R. Statistical language learning: mechanisms and constraints. Current Directions in Psychological Science, 12(4), 110-114.

Saffran et al. 1996: Saffran, J. R., Aslin, R. N., Newport, E. L. Statistical Learning by 8-Month-Old Infants. Science, 274(5294): 1926-1928.

Sebastian Galles et al., 2012: Sebastian Galles, N., Albareda-Castellot, B., Weikum, W., Werker, J. A Bilingual Advantage in Visual Language Discrimination in Infancy. Psychological science, 23, 994-9. <https://doi.org/10.1177/0956797612436817.>

Thal, Bates 1988: Thal, D., Bates, E. Language and gesture in late talkers. Journal of Speech and Hearing Research 31, 115-123.

Tomasello et al. 2007: Tomasello, M., Carpenter, M., Liszkowski, U. A New Look at Infant Pointing. Child Development, 78, 705-722.

Tomblin et al. 1997: Tomblin, J. B., Records, N. L., Buckwalter, P., Zhang, X., Smith, E., $\mathrm{O}$ ‘Brien, M. Prevalence of specific language impairment in kindergarten children. Journal of speech, language, and hearing research: JSLHR, 40(6), 1245-1260.

Trecca et al. (in press)a: Trecca, F., Bleses, D., Højen, A., Madsen, T.O., Christiansen, M.H. When too many vowels impede language processing: An eye-tracking study of Danishlearning children. Speech and Language.

Trecca et al. (in press)b: Trecca, F., Tylén, K., Højen, A., Christiansen, M. H. Danish as a window onto language processing and learning. Language Learning. 
Vulchanova et al. 2011: Vulchanova, M., Vulchanov, V. Stankova, M. Idiom comprehension in the first language: a developmental study. VIAL 8, 141-163.

Vulchanova et al. 2014: Vulchanova, M., Foyn, C. H., Nilsen, R. A., Sigmundsson, H. Links between phonological memory, first language competence and second language competence in 10-year-old children. Learning and individual differences. vol. 35.

Vulchanova et al. 2015: Vulchanova, M., Saldaña, D., Chahboun, S., Vulchanov, V. Figurative language processing in atypical populations: the ASD perspective. Frontiers in Human Neuroscience, 9, 24. <https://www.frontiersin.org/article/10.3389/fnhum>

Vulchanova et al. 2017: Vulchanova, M., Baggio, G., Cangelosi, A. \& Smith, L. Editorial: Language Development in the Digital Age. Frontiers in Human Neuroscience, 11, 447.

Vulchanova et al. 2019: Vulchanova, M., Chahboun, S., Galindo-Prieto, B., Vulchanov, V. Gaze and Motor Traces of Language Processing: Evidence from Autism Spectrum Disorders in Comparison to Typical Controls. Cognitive Neuropsychology, 36:7-8, 383-409.

Vulchanova et al. 2020: Vulchanova, M., Saldaña, D., Baggio, G. Word structure and word processing in developmental disorders. - In: Pirrelli, V., Plag, I., Dressler, W (Eds.). Word Knowledge and Word Usage (Chapter 15: Berlin, Boston: De Gruyter Moton. 680-708.

Weikum et al. 2007: Weikum, W., Vouloumanos, A., Navarra, J., Soto-Faraco, S., Sebastian Galles, N., Werker, J. Visual Language Discrimination in Infancy. Science, 316. 1159. 10.1126/science. 1137686.

Werker, J., Byers-Heinlein, K. 2008: Bilingualism in infancy: First steps in perception and comprehension. Trends in Cognitive Science, 12,144-151.

Yee et al. 2012: Yee, M., Jones, S., Smith, L. Changes in Visual Object Recognition Precede the Shape Bias in Early Noun Learning. Frontiers in Psychology, 3, 533.

Özçaliskan et al. 2016: Özçaliskan, S., Adamson, L. B., Dimitrova, N. Early deictic but not other gestures predict later vocabulary in both typical development and autism. Autism, 20(6), 754-763.

\section{Useful links to on-line material:}

The child's mind: https://www.ted.com/talks/patricia_kuhl_the_linguistic_genius_of_babies Early word production: https://www.ted.com/talks/deb roy the birth_of_a_word Word learning: https://bold.expert/why-do-children-learn-the-words-they-do/

Child-Directed Speech: https://www.sciencealert.com/amotheresea-helps-kids-speak

Language Impairment: http://deevybee.blogspot.com/2013/09/raising-awareness-oflanguage-learning.html

Early Language Development in the Digital Age (large-scale Horizon2020 project): https:// www.ntnu.edu/e-ladda/

Mila Vulchanova, Valentin Vulchanov

Language Acquisition and Language Processing Lab

Norwegian University of Science \& Technology - Norway

Acknowledgements

Work on this project has received funding from the European Union's Horizon 2020 research and innovation programme under the Marie Sklodowska-Curie Actions grant agreement No 857897 